\title{
El derecho cooperativo y el docente cooperativo
}

(Cooperative law and cooperative teacher)

\author{
José Alberto Yorg y y Ana María Ramírez Zarza² \\ TECNICOOP (Argentina)
}

Sumario: I. Introducción. II. Marco conceptual del problema. III. Método de investigación. IV. Conclusión. V. Bibliografía y otras fuentes.

Summary: I. Introduction. II. The Conceptual framework of the problem. III. The Research Method. IV. Conclusion. V. Bibliography and other sources.

Resumen: La falta de un marco regulatorio claro y completo para los/as docentes cooperativos/as, trabajadores de la educación cooperativa en el ámbito formal del sistema educativo, como especialidad o modalidad específica y reconocida por ley, constituyen una de las principales causas de preocupación y cuya ocupación es reflejada por este artículo.

Esto plantea, con todo rigor, un estudio exaustivo para luego impulsar con vigor necesario la necesidad de la creación de la figura legal del «docente cooperativo». Corresponde, a nuestro juicio, al Derecho cooperativo dar los primeros pasos en la consecución de ello. Al igual que el legislador curioso observa determinada actividad humana y la plasma en una normativa que la regula y la legaliza, tal procedimiento puede alcanzar la creación de la figura del «docente cooperativo».

Palabras clave: Derecho Cooperativo. Docente cooperativo. Educación Cooperativa Escolar y Universitaria.

Abstract: The lack of a clear and complete regulatory framework for cooperative teachers, cooperative education workers in the formal sphere of the education system, as a specific specialty or modality and recognized by law, constitute one of the main causes of concern and whose occupation is reflected by this article.

This raises, with all rigor, an exhaustive study and then vigorously promote the need for the creation of the legal figure of the «cooperative teacher». It

1 Profesor. Licenciado en Administración. Especialista en Políticas Socioeducativas. Email: tecnicoop@yahoo.com.ar

2 Profesora. Contadora. Licenciada en Administración. Especialista en Políticas Socioeducativas. Email: tecnicoop@yahoo.com.ar 
corresponds, in our opinion, to the cooperative right to take the first steps in achieving it. Just as the curious legislator observes certain human activity and the plasma in a regulation that regulates and legalizes it, such a procedure can reach the creation of the figure of the "cooperative teacher».

Keywords: Cooperative Law Cooperative teacher Cooperative School and University Education. 
«Hace 48 años desde 1964 cuando la cultura solidaria fue plasmada en la eficacia de la Ley N' 16.583 que institucionalizó una formal educación cooperativa, declarando de alto interés nacional la enseñanza de los principios del cooperativismo, obligando al estado a dictar las normas para la inclusión de los planes y programas de los establecimientos educacionales de su dependencia, de la enseñanza teórico-práctica del cooperativismo e interesar a los gobiernos de las provincias su implantación en los establecimientos educacionales».

Dr. Roberto Fermín Bertossi.(2012).

Nosotros agregamos: Sin embargo, no se estableció la figura del «docente cooperativo».

\section{Introducción}

El trabajo que presentamos es producto de un estudio sobre la temática, pero fundamentalmente proviene de nuestra larga experiencia en la materia, y por tanto, nos interesa convocar a poner la mirada sobre la misma, acercar la importancia de la formación y reconocimiento de crear la figura del docente o del profesor cooperativo en la implantación del proceso enseñanza-aprendizaje en todas las modalidades y niveles del sistema oficial educativo.

Los resultados obtenidos de nuestra reflexión y analisis nos permiten indicar que la importancia adquiere alto nivel de proyección en el avance institucional cooperativo, esperando que en la comunidad científica cooperativa este tema vaya a alcanzar interés y ayuden con ello a solucionar la problemática relacionada con los aspectos académicos y técnicos y de interacción social.

\section{Marco conceptual del problema}

Para una mejor comprensión de cuanto abordamos aquí, en referencia al docente en la Ley de educación nacional argentina (26206) en sU CAPITULO II LA FORMACION DOCENTE

ARTICULO 71. - La formación docente tiene la finalidad de preparar profesionales capaces de enseñar, generar y transmitir los co- 
nocimientos y valores necesarios para la formación integral de las personas, el desarrollo nacional y la construcción de una sociedad más justa. Promoverá la construcción de una identidad docente basada en la autonomía profesional, el vínculo con la cultura y la sociedad contemporánea, el trabajo en equipo, el compromiso con la igualdad y la confianza en las posibilidades de aprendizaje de los/as alumnos/as. ( El subrayado es nuestro)

ARTICULO 73. - La política nacional de formación docente tiene los siguientes objetivos: b) Desarrollar las capacidades y los conocimientos necesarios para el trabajo docente en los diferentes niveles y modalidades del sistema educativo de acuerdo a las orientaciones de la presente ley. e) Incentivar la investigación y la innovación educativa vinculadas con las tareas de enseñanza, la experimentación y sistematización de propuestas que aporten a la reflexión sobre la práctica y a la renovación de las experiencias escolares.

Enmarcamos lo anterior en el camino de otorgar razonamiento y argumentación sobre el ARTICULO 90. - El Ministerio de Educación, Ciencia y Tecnología promoverá, a través del Consejo Federal de Educación, la incorporación de los principios y valores del cooperativismo y del mutualismo en los procesos de enseñanza-aprendizaje y la capacitación docente correspondiente, en concordancia con los principios y valores establecidos en la Ley N. ${ }^{\circ} 16.583$ y sus reglamentaciones. Asimismo, se promoverá el cooperativismo y el mutualismo escolar.

El Derecho cooperativo argentino no alberga en su plexo una categoría como la de «Docente cooperativo» o similares.

Esta realidad implica que se realicen todos los esfuerzos necesarios para lograr esa categoría profesional educativa, pues ello significaría un gran paso adelante en la estructuración pedagógica cooperativa en todos los niveles y modalidades del sistema oficial educativo, sólidamente.

Que sin duda alguna este planteamiento demuestra la conveniencia vital de instalar tal figura educativa profesional que llevaría a ganar y cubrir posiciones institucionales con su consecuente incidencia educativa y política.

Aquí evidenciamos que el Derecho cooperativo no se desenvuelve sólo o fuera de los conflictos sociales, económicos y políticos. En efecto, el Derecho cooperativo interactua con el Derecho laboral y derechos de incidencia social en las sociedades concretas y por ello abordamos estas particularidades.

Nos lo dice el Dr. León SCHUJMAN (1986) «... hoy la complejidad de la realidad, obliga a la academia a pensar en la pluridisciplinariedad y en la ecología de saberes, que reclama Boaventura Da Souza San- 
tos, y son esos saberes que engendra la praxis, los que mejor explican dónde están las injusticias que reclaman de la justicia que se comporte como talı.

Sin duda, Schujman, al leer nuestro trabajo «Interrelación entre el derecho laboral, derecho de incidencia colectiva y el derecho cooperativo. Estudio de caso» (YORG y RAMíREZ ZARZA 2018), acuerda con el enfoque que le imprimimos a la investigación y conclusión arribada de que al Derecho cooperativo corresponde estudiarlo y enriquecerlo desde la pluridisciplinariedad, desde la interrelación, con otros derechos».

Es en esa visión que planteamos y proponemos que el Derecho cooperativo debe evolucionar, debe modernizarse, para ser capaz, juridicamente, de demostrar y lograr sentar jurisprudencia, sin ambages, por ejemplo, que la educación cooperativa escolar y el/la docente cooperativo/a forman parte indisoluble del sistema de educación formal argentino.

No se parte de cero para tal avance, particularmente nosotros, docentes cooperativos, hemos realizado un documento que puede ser materia prima y se conoce como «Alegato pedagógico cooperativo. Bases pedagógico-político-jurídicas de una petición de justicia».

Por otra, desde el Estado nacional argentino, se tienen fuentes concretas desde el año de 1954, 1961 y 1986, entre otros. En la Provincia de Formosa, sucesivamente 1971, 1985 y 1995. En organismos específicos oficiales de educación cooperativa escolar se anota, con ausencia del perfil o categoría del docente cooperativo.

De cualquier escalón de la enseñanza oficial —pre-primaria, primaria, secundaria, terceria y universitaria-, podríamos esbozar un perfil de la o del docente cooperativo, con los riesgos que ello conlleva, como aquella persona que puede acreditar solvencia tecnica-pedagógica y didáctica del cooperativismo, con fuerte compromiso doctrinario, con independencia de otros titulos de grado que posea, ante quien corresponda.

\section{Método de investigación}

Nuestra tesis pedagógica se funda a la luz de los principios científicos que implican el indagar al fenómeno de estudio y explicar las leyes de funcionamiento que informan sobre él. Así, con ese razonamiento elaboramos nuestra ponencia "Génesis de la Pedagogía cooperaria» presentada ante el «VI Encuentro de investigadores Latino-Américanos en Cooperativismo», realizado en San Lorenzo (Paraguay) los días 13, 14 y 15 de octubre de 2010 (YORG 2010). 
Como no escapa a nadie, el Derecho cooperativo lucha todavía por el reconocimiento de su autonomía científica definitiva, y en función a ello, realiza grandes esfuerzos para obtener un método propio que lo independice totalmente de las tendencias jurídicas liberales y romanistica que le nublan del objeto específico que le ocupa, de su esencia propia, cual es la cooperación en función normativa.

Reiteradamente recurrimos al maestro Leon SCHUJAM (1985) por la solvencia de sus escritos y por el afecto que nos une a su recuerdo de persona con sencillez de los grandes:

«El método es el camino que sigue el pensamiento para conocer la realidad. Otro elemento esencial para tener en cuenta en el enfoque cooperativo de la realidad es que el cooperativismo nace y se desarrolla históricamente como un movimiento de cambio y progreso social.

Nacido como respuesta crítica a la injusticia social a que condujo el desarrollo del capitalismo y su posterior expresión de la concentración monopólica, su comportamiento ha sido solidario con las causas progresistas de la humanidad. Sin embargo, está en abierta contradicción con la mayor parte del mensaje explícito y subliminal que el sistema educativo y los medios de comunicación social, trasmiten en concordancia con los intereses del sistema económico vigente y sus usufructuarios directos.

El medio económico social en que la cooperativa se inserta es antagónico a su existencia. Los hombres y mujeres que la integran son producto de ese medio y sufren su permanente influencia. La conducta cooperativa supone un comportamiento no sólo distinto sino también consciente de la necesidad de cambio».

De estas conceptualizaciones extraidas nos valdremos, metodológicamente, para avanzar en el encuadre de nuestra concepción sobre la temática en estudio y su exposición.

Consideramos, modestamente, que de lo que se trata es de explicar las causas por las cuales los fenómenos, en este caso el fenomeno «cooperación», en función de movimiento socio-económico, se manifiestan de una forma, es decir, poseen esencia peculiar que el investigador descubre y lo explica. El legislador observó este fenomeno y lo plasmó legislativamente en orden al «acto cooperativo».

El método de indagación consiste, entonces, en buscar la esencia peculiar de las cosas o de los fenómenos sociales superando la mirada profana o de observar sólo sus apariencias externas.

Así pues, tomando como base y sutento lo consigando más arriba del legado de Don León, nos interrogamos, ¿cómo establecer las com- 
petencias del título?, ¿cómo determinar los perfiles de las correspondientes incumbencias y alcances y orientación para el ejercicio profesional del docente cooperativo?

Pensamos que existen inumerables experiencias, en su más variada forma y manera de aplicación en todo el territorio argentino que, avalados en normativas nacionales y provinciales, se han llevado a cabo y en su mayoría con éxito. Por lo que no resultaría una labor fatigosa para los organismos de Educación Técnico-pedagógico y Profesional definir tales aspectos, al contrario, sería de enorme vitalidad en cuanto a conocer formidables aportes pedagógicos innovadores de bien social.

El servicio público de la educación cooperativa escolar por parte del Estado es de inexcusable cumplimiento por intervención constiucional y legal. Ello habilita entonces a formular la creación de la figura del "docente cooperativo» con miras a perfeccionar esas normativas, ayudando precisamente al Estado al fiel cumplimiento de esa responsablidad.

Robert OWEN nos presentó con toda crudeza la imposibilidad de que la educación común tenga su efecto positivo sin tener su correlato en una nueva forma de organizar la sociedad, al decirnos «En mi calidad de empleador y director de manufactura en Lancashire y Lanarkshire, hice todo lo que pude para aliviar los males de mis empleados; y sin embargo, a pesar de todo lo que hice, con nuestro sistema totalmente irracional de creación de riqueza, de formación del carácter y de organización de todas las actividades humanas, sólo pude aliviar un poco la miseria de su estado; y ello pese a ser consciente de que la sociedad, incluso entonces, poseía medios sobrados para educar, emplear y gobernar a la población entera del Imperio Británico, haciendo de ellos hombres formados e inteligentes, unidos y prósperos para siempre, y para convertirlos en hombres y mujeres felices, de cualidades físicas y mentales superiores». (OWEN, 1858).

Hecho histórico constatado por nosotros más de un siglo después al actuar como maestros de escuelas rurales de la Provincia de Formosa, al ver paulatinamente nuestros esfuerzos educativos disgregarse en la nada, pues en las campiñas formoseñas los cultivos tradicionales sólo generan mayor pobreza y desesperanza, ya que la educación común no brinda los conocimientos empresariales emancipatorios requeridos, quedando ella consecuentemente, casi descolgada de esa realidad. Esa realidad nos llevó, inspirados por el estimulo de una supervisora escolar, a estudiar la carrera universitaria de tecnicatura en cooperativismo.

El modelo productivo cooperativo, surgido como respuesta al naciente capitalismo industrial, se materializa en una empresa de propie- 
dad cooperativa que humaniza la economía basada en una concepción diferente de la organización de la sociedad y el hombre. Esa diferencia no es un dato menor, pues implica valores y principios fraternales y equitativos en las relaciones sociales que aniquilan antagonismos. "Ordenar la organización de la producción de tal modo que permita dar a cada cual las condiciones materiales y espirituales para gozar de la libertad y de la igualdad», nos dijo el pensador ROUSSEAU (1975), y es pertinente recordarlo permanentemente. La pedagogía cooperativa debe ser, necesariamente, reflejo y fundación de esa concepción.

Y si asumiéramos, plenamente, como nos inculca Enrique AGILDA (1962), en su magnifico libro «Cooperación, doctrina de armonía» podríamos preguntarnos si esta cualidad hermosa es también intrínseca a su Pedagogía y diríamos sin dilación que sí, ¿Por qué? Porque la cooperación se expresa en su dimensión pedagógica proveyendo conocimientos y estímulos "para sentir y pensar a favor de la convivencia armónica con sus semejantes».

Aporta también "conocimientos de los aspectos negativos que impiden el progreso» armónico social, económico de los pueblos. De tal forma a que «en razón de ese conocimiento el cooperante trabaja con fe inquebrantable y renovado empeño por superar los males».

Del mismo modo encontramos huellas inequívocas de la orientación pedagógica cooperativa al leer atentamente los objetivos de la idea de la Cooperación que tuvieron los Pioneros, pues destacaban la imperiosa necesidad de construir un mundo con una nueva moral.

Tal era la idea expuesta en el primer Manifiesto en que difundían los ideales de la verdadera cooperación, destacaban la gran necesidad de construir, lenta pero ininterrumpidamente, un mundo con una nueva moral y un mejor sistema de sociedad, nos narra Goedhart, presidente de la ACl-1921-1927. Sigamos con el maestro GOEDHART (1995) cuando nos ilustra sobre los Pioneros de Rochdale en sus inicios «tenían tras ellos la terrible experiencia de las condiciones de vida y trabajo que habian prevalecido durante y después del periodo de la revolución industrial. Eran conscientes, por experiencia, que los más pobres de los pobres eran esquilmados por los intermediarios quienes les cobraban precios de usurero para entregarles productos adulterados, robarles en el peso y extorsiónalos con los precios a cambio de sus miserables salarios.»

No podemos evitar el preguntar tras la lectura del párrafo anterior ¿no describe nuestro buen amigo una realidad también actual?

Así comprenderemos porque la Pedagogía Cooperativa es contestataria a «la moral perversa que empujó a los Pioneros y les dio fuerzas y coraje para empezar a luchar contra esas condiciones adversas» (GOEDHART 1995). «Esta fue también la razón por la que se esforzaron 
por analizar las causas de las injusticias económicas en las que vivían y buscar los medios de terminar con ese viejo orden perverso, atacándolo en sus causas». i i En extraordinaria luz Goedhart nos iluminó la génesis de la pedagogía cooperativa!!

$Y$ es que el Derecho y la educación cooperativa no se desarrollan y funcionan-como todos seguramente querríamos-de manera absolutamente autónomos de la economía dominante, la capitalista. Actualmente en su faz financiera, se encuentran ambos campos del cooperativismo de manera condicionados, si bien, como disciplina científica la pedagogía y el Derecho cooperativo poseen autonomía.

Ambos saberes y sus actores, el jurista cooperativo y el educador cooperativo, son factores fundamentales para el propio desarrollo de la cooperación en el mundo y conlleva también, un factor preponderante en el proceso de transformación pacífica y democrática de la sociedad.

\section{Conclusiones}

Al igual que el legislador curioso observa determinada actividad humana y la plasma en una normativa que la regula y la legaliza, sin embargo, en el caso de la cooperación, ese curioso legislador tendrá previamente que comprender, y en este punto, confesamos que quisimos extremar cuánto aludimos, indagamos y nos encontramos con un vídeo explicativo sobre el término «comprender» por parte de José Ángel ACHÓN INSAUSTI (2016).

Nos dice, entre otros, que "comprender» significa "penetrar en las motivaciones que llevan a las personas y grupos concretos a actuar de tal forma, a su mentalidad y a la cultura, a esas motivaciones de fondo que los mueven». En el caso del cooperativismo, el legislador debe previamente comprender las motivaciones profundas de la esencia de la cooperación para plasmarlo en normas.

Tal cual sucedió históricamente con la actividad empresarial cooperativa, el educador inquieto debe también desentrañar las leyes internas del funcionamiento del fenómeno «cooperación», es decir, los mecanismos que explican el comportamiento del «hecho cooperativo» en su faz educativa y proporcionar, a partir de los hallazgos, los principios pedagógicos inherentes a él. Es nuestro reto.

Pero bueno, dejemos hablar en este punto particular a Paúl LAMBERT (1975): "La ciencia explica lo real, la doctrina juzga y propone diversos cambios para mejorar lo real». Y nos profundiza ese pensamiento «en lo que se refiere a la cooperación, la doctrina precede a la ciencia al preceder también al propio hecho cooperativo: la coope- 
ración ha surgido de un juicio que los fundadores hicieron sobre el mundo tal y como se les presentaba inmediatamente después de la revolución industrial, y la cooperación ha nacido precisamente porque este juicio era desfavorable».

Nos narra LAMBERT (1975) como en su día dijo Charles Gide, la cooperación es a la vez objeto de ciencia y razón de vivir. Por ello, su estudio completo implica también un estudio científico y un estudio doctrinal. Es lo que estamos tratando de hacer.

Abreviando, corresponde a la Doctrina y a la Filosofía Cooperativa como nos dice LAMBERT (1975) «juzgar y proponer» lo que se debe enseñar, para que sea la pedagogía y la didáctica cooperativa la que diga cómo se debe enseñar.

La Pedagogía Cooperativa y su didáctica constituyen el método de la reproducción intelectual de la realidad e identidad cooperativa. Es, en definitiva y específicamente, la explicación del fenómeno económico-social cooperativo en términos de enseñanza-aprendizaje, partiendo de la actividad empresarial objetiva del hombre cooperativo en contravención a la ley del mercado en que está cercado. Somete a discusión los problemas acerca de la forma y objetivos de la producción del conocimiento parado desde una posición doctrinaria de armonía.

Derecho cooperativo y la educación cooperativa son expresiones en campos específicos de acción en la sociedad del cooperativismo. Ello habilita, a nuestra modesta opinión, definir el actor impulsor: el docente y/o profesor cooperativo, regularmente reconocido por la normativa pertinente.

Hablamos en definitiva del docente cooperativo - como categoría profesional y laboral - con el objeto lograr el reconocimiento efectivo de los derechos y obligaciones laborales y profesionales de aquellos que están ejerciendo hoy y de los que vendrán, en aras del mejoramiento de la calidad de la enseñanza, la inclusión educativa, la investigación y la extensión cooperación educacional.

\section{Bibliografía y otras fuentes}

AGILDA, Enrique. 1962. Cooperación, doctrina de armonía. 2. edición. Buenos Aires: Intelcoop.

ANDER EGG, Exequiel. 1989. Técnicas de investigación social. 21. a edición. Buenos Aires: Humanitas.

ARNÁEZ ARCE, Vega María. 2018. «La alternativa cooperativa en la prestación de servicios públicos sostenibles». Boletín de la Asociación Internacional de 
Derecho Cooperativo 52: 123-135. doi: http://dx.doi.org/10.18543/baidc52-2018pp123-135.

BERTOSSI, Roberto Fermín. 2012. «La educación cooperativa es ley». El Cronista. 7 de mayo de 2012.

GAMINDE EGIA, Eba y MARTÍNEZ ETXEBERRIA, Gonzalo. 2019. «Training of cooperative values as a decisive element in new jobs to be created by 21st century cooperatives». Boletín de la Asociación Internacional de Derecho Cooperativo 54: 97-114. doi: http://dx.doi.org/10.18543/baidc-542019pp97-114.

GOEDHART, G.J.D.C. 1995. «El aspecto moral de la cooperación». Revista de la Cooperación Internacional, volumen 28, n. $^{\circ} 2$.

HERNÁNDEZ ARTEAGA, Isabel; PÉREZ MUÑOZ, Colombia; RUA CASTAÑEDA, Sohely. 2018. «Intereses y perspectivas formativas en Economía Social y Solidaria de los estudiantes universitarios», CIRIEC-España, Revista de Economía Pública, Social y Cooperativa 94: 91-121. doil: 10.7203/CIRIECE.94.12782.

LAMBERT, Paúl. 1975. La Doctrina Cooperativa. 4. a edición. Buenos Aires: Intercoop.

LONDOÑO, Carlos Mario. 1976. Movimiento Cooperativo y el Estado. Buenos Aires: Intercoop.

MARTÍNEZ-LEÓN, Inocencia; OLMEDO-CIFUENTES, Isabel; ARCAS-LARIO, Narciso; ZAPATA-CONESA, Juan. 2018. "Las cooperativas en la educación: satisfacción laboral del profesorado y diferencias de género», CIRIEC-España, Revista de Economía Pública, Social y Cooperativa 94: 31-60. doi: 10.7203/CIRIECE.94.12700.

MELIÁN, Amparo; CAMPOS, Vanessa; SANCHIS, Joan Ramón. 2017. "La educación de postgrado en Economía Social en la universidad española ¿una asignatura pendiente?», CIRIEC-España, Revista de Economía Pública, Social y Cooperativa 89, 33-54.

MORENO FONTELA, Juan Luis. 2017. "Las relaciones entre los valores y principios cooperativos y los principios de la normativa cooperativa». REVESCO. Revista de Estudios Cooperativos 124: 114-127. doi: http://dx.doi.org/10.5209/ REVE.54923.

OWEN, Robert. 1858. The life of Robert Owen: written by himself [La vida de Robert Owen por él mismo]. Vol. 1. Londres: Effingham Wilson.

PANZONI, Erico E. 1959. «Funciones del Estado en sus relaciones con el Cooperativismo» Revista del Instituto de Estudios Cooperativos. Enero-Febrero-Marzo 1959. N. ${ }^{\circ} 3$.

ROUSSEAU, Jean-Jacques. 1975. El contrato social. Madrid: Austral.

SÁNCHEZ ESPADA, Javier; MARTíN LÓPEZ, Sonia; BEL DURÁN, Paloma; LEJARRIAGA PÉREZ DE LAS VACAS, Gustavo. 2018. «Educación y formación en emprendimiento social: características y creación de valor social sostenible en proyectos de emprendimiento social». REVESCO. Revista de Estudios Cooperativos 129: 16-38. doi: http://dx.doi.org/10.5209/REVE.62492.

SCHUJMAN, León. 1986. «La Enseñanza del Cooperativismo en la Educación Pública». Revista de Idelcoop, Volumen 13, № 48. 
SCHUJMAN, León. 1985. «El método de análisis y el contenido teórico del enfoque cooperativo». Revista de Idelcoop, Volumen 12, N. $^{\circ} 47$.

YORG, José Alberto. 2010. "La génesis de la Pedagogía Cooperativa». REDDOLAC Red de docentes de América Latina y del Caribe. https://reddolac.org/forum/ topics/ponencia-la-genesis-de-la

YORG, José Alberto y RAMÍREZ ZARZA, Ana María. 2018. «Interrelación entre el derecho laboral, derecho de incidencia colectiva y el derecho cooperativo. Estudio de caso» Boletin de la Asociacion Internacional de Derecho Cooperativo 53: 275-288. doi: http://dx.doi.org/10.18543/baidc-53-2018pp275-288.

YORG, José Alberto y RAMíREZ ZARZA, Ana María. 2019. «Propuesta didáctica para la enseñanza del cooperativismo escolar rural». Portal del Ministerio de Educación Nacional en su capítulo denominado Mapa Educativo Nacional en la especialidad de Educación Rural. www.mapaeducativo.edu.ar.

\section{Otras fuentes documentales}

ACHÓN INSAUSTI, José Angel. 2016. ¿Qué significa comprender?. Video localizado en el siguiente link: https://www.youtube.com/watch?v=09Qtg0DUsUo.

Codigo Civil y Comercial de la Nación-Ley 26.994/ 2014

Constitución de la República Argentina

Ley de Cooperativas 20337

Ley 23427 y 744 Fondo de Educación y Promoción Cooperativa

Ley de Educación Nacional N²6 206/06 


\section{Derechos de autor}

El Boletín de la Asociación Internacional de Derecho Cooperativo es una revista de acceso abierto lo que significa que es de libre acceso en su integridad inmediatamente después de la publicación de cada número. Se permite su lectura, la búsqueda, descarga, distribución y reutilización legal en cualquier tipo de soporte sólo para fines no comerciales y según lo previsto por la ley; sin la previa autorización de la Editorial (Universidad de Deusto) o el autor, siempre que la obra original sea debidamente citada (número, año, páginas y DOI si procede) y cualquier cambio en el original esté claramente indicado.

\section{Copyright}

The International Association of Cooperative Law Journal is an Open Access journal which means that it is free for full and immediate access, reading, search, download, distribution, and lawful reuse in any medium only for non-commercial purposes, without prior permission from the Publisher or the author; provided the original work is properly cited and any changes to the original are clearly indicated. 